\title{
MONITORING PRICE TAG WITH EPAPER BY USING 6LoWPAN AND
}

\section{MQTT}

\author{
Kapuluru Chenchaiah', BVNR. Siva Kumar², P. Rakesh Kumar ${ }^{3}$ \\ ${ }^{1}$ M.Tech Scholar, ECE Department, Lakireddy Bali Reddy College of Engineering, Andhrapradesh, India \\ ${ }^{2}$ Associate Professor, ECE Department, Lakireddy Bali Reddy College of Engineering, Andhrapradesh, India \\ ${ }^{3}$ Sr.Asst.Professor, ECE Department, Lakireddy Bali Reddy College of Engineering, Andhrapradesh, India
}

\begin{abstract}
Internet of things (IoT) is an advanced automation and analytics system which exploits networking, sensing, big data, and artificial intelligence technology to deliver complete systems for a product or service.iot systems have the ability to suite in any environment and have applications across industries due to their unique flexibility. They improve the quality of data collection automation and much more through smart devices and powerful enabling technology.
\end{abstract}

One of the open source operating system for the internet of things (IoT) is Contiki. This is used to connect low power microcontrollers and tiny low cost to the internet.

As a sub-domain, "Wireless price tag with customer survey" Networks have been attracting a lot of use in daily super markets and shopping malls. The resource is for to reduce time for workers and easy to update daily price and data for a particular product. In this project the "PHYNODE-EPAPER" with 2 sensors, 1 is the temperature sensor and 2 is the proximity sensor is developed and implemented. Our main aim is also to reduce the power consumption. The tool is applicable to 6LoWPAN and MQTT technology.

Keywords: IoT, 6LoWPAN, PHYNODE-ePAPER, MQTT

\section{INTRODUCTION}

There is an increasing interest in using wireless communication with sensors and actuators in homes, office buildings factories, and even outdoors. Moreover, there is a desire to incorporate these devices as part of the internet so that these devices could be accessed from anywhere.

From this perspective, embedding a TCP/IP stack into these sensing and acting devices seems an attractive idea, which is reinforced by the new features IPv6 provides (such as the large address space and address auto configuration). However, the TCP/IP protocol suite was not originally intended for such devices; its requirements for the underlying link layers are generally too strong to be carried out by resource-constrained devices, while certain network layer features are too complex and resource consuming.

This project mainly deals with the drivers for all the sensors present on the Phynode i.e CC2650 PhyWave in contiki OS and then make an application which sends the value of all the sensors to the Phygate (Gateway or edge router) i.e.AM 335xPhytech Wega Board. This application sends the data using 6LoWPAN.

The below sections describes about phynode and ePaper in details

\subsection{Wireless Sensor Network (WSN)}

Wireless sensor is also called as wireless sensor and actuator networks. This is built of nodes. Many applications such as detecting and tanks on a battle ground, tracking personnel in buildings etc uses wireless sensor networks.

\section{INTERNET OF THINGS (IoT)}

IoT is the short form of internet of things. IoT stands for interconnection of internet enabled devices or things.

There are many features of IoT. Some of the features are artificial intelligence, sensors and small device use.

\subsection{How it Works}

IoT consists of all the web enabled devices that collect, send and act on data they acquire from their surrounding environments using embedded sensors, processors and communication hardware.

The hardware utilized in IoT systems includes devices for a remote dashboard, devices for control, servers a routing or bridge device and sensors. 


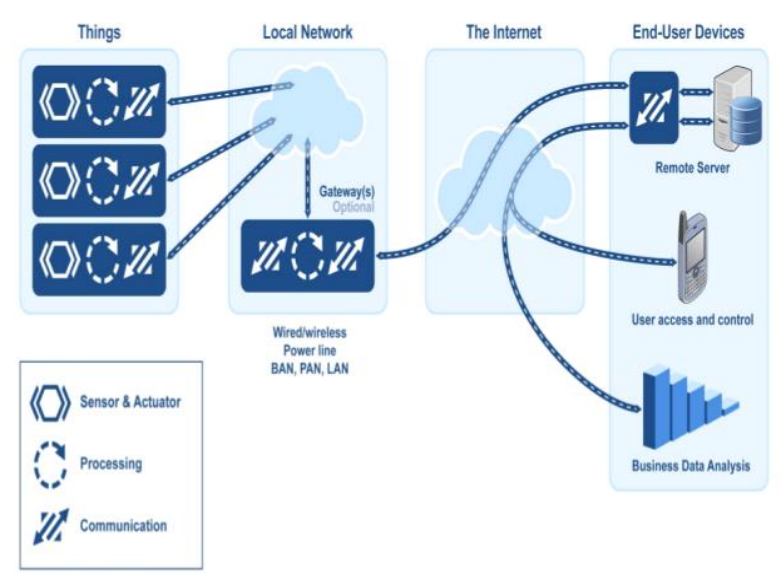

Fig-1 General set up of IOT

\subsection{The Internet Protocol Suite}

This is commonly referred as TCP/IP. This is used on the internet and computer networks. The functionality is offered in four layers which are used to sort all related protocols. The layers are as follows

$>$ Application layer.

$>$ Transport layer

$>$ Internet layer

$>$ Link or physical layer

The below figure shows the block diagram of the TCP/IP with different layers.

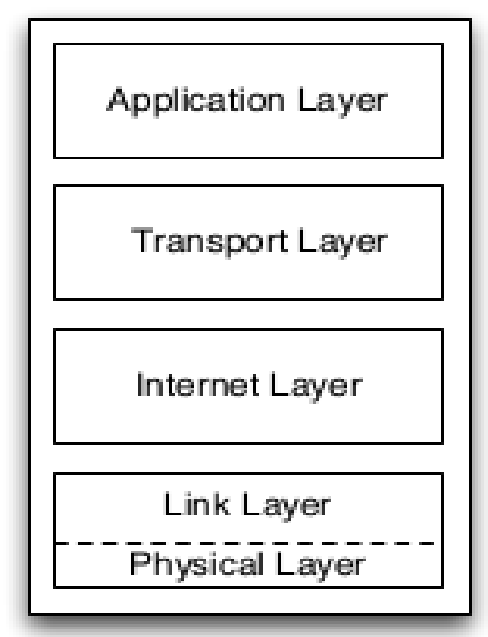

Fig.-2 The TCP/IP stack, including the Physical Layer (dashed)

\subsection{Internet Protocol}

Internet protocol defines the addressing methods that are used to label the datagram with source and destination information. It is used to send or deliver the packets from the source to the destination based on the IP addresses in the packet headers

\subsubsection{IPV4}

The first version of the protocol to be widely deployed is the IPV4 and it is the fourth version of the internet protocol.
Internet protocol being a layer-3 protocol (OSI) takes data segments from layer-4(transport) and divides it into packet. IP encapsulates data unit received from above layer and adds its own header information.

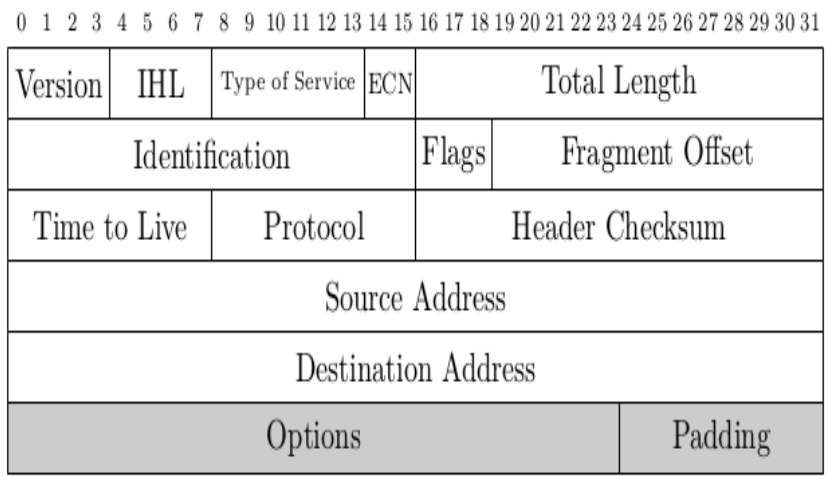

Fig- 3 IPv4 Datagram Header. Light Grey Coloured Fields Are Optional

\subsubsection{IPv6}

Internet protocol version 6 is the most recent version of the internet protocol (IP) that provides identification and location system for computers on networks.

Internet Protocol version 6 is a new addressing protocol designed to incorporate all the possible requirements of future Internet known to us as Internet version 2. This protocol as its predecessor IPv4, works on the Network Layer (Layer-3). Along with its offering of an enormous amount of logical address space, this protocol has ample features to which address the shortcoming of IPv4

\begin{tabular}{|c|c|c|c|}
\hline Version & Traffic Class & \multicolumn{2}{|c|}{ Flow Label } \\
\hline \multicolumn{2}{|r|}{ Payload Length } & Next Header & Hop Limit \\
\hline \multicolumn{4}{|c|}{ Source Address } \\
\hline \multicolumn{4}{|c|}{ Destination Address } \\
\hline
\end{tabular}

Fig. 4 IPv6 Datagram Header

\section{6LoWPAN}

6LoWPAN is an intermediate layer that allows the transport of ipv6 packets over IEEE 802.15 frames. The abbreviated 6 LoWPAN is IPV6 over low power wireless personal area networks. The below figure shows the intermediate layer of 6 LoWPAN. 


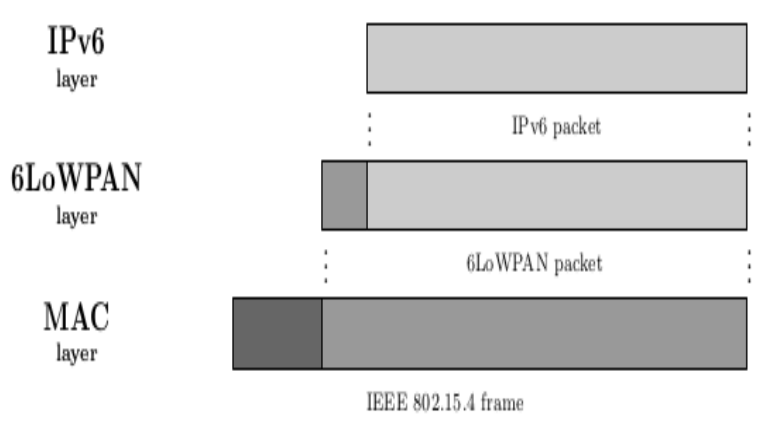

Fig- 5. 6LoWPAN Intermediate layer

\subsection{LoWPAN Motivations}

To avoid the fragmentation as much as possible, 6LoWPAN defines an IPV6 header compression mechanism. 6LoWPAN does not use large packets.

6LoWPAN layer is in between data ink layer and original Network layer.

\section{HARDWARE}

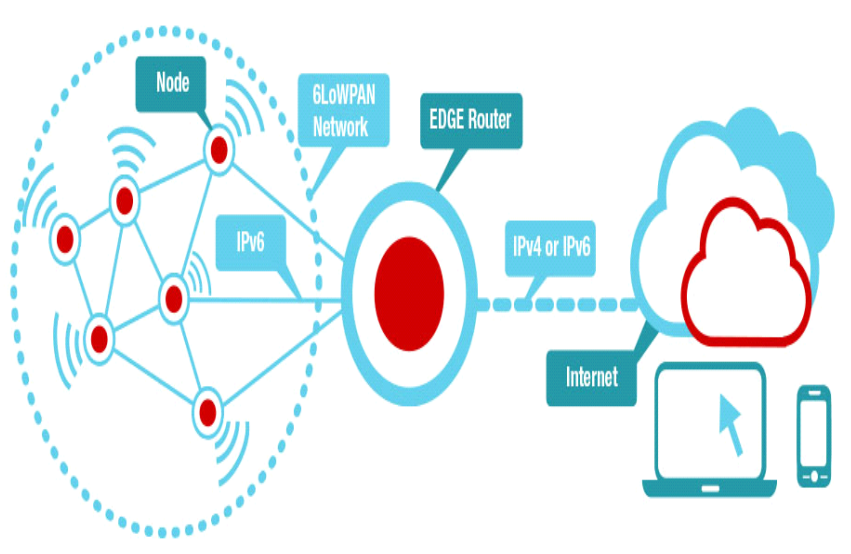

Fig-6 General hardware setup

\subsection{Phynode}

Phynode serves as platform for different wireless communication modules which can be mounted via DSC (Direct Solder Connection). The phynode board is equipped with different sensors to perceive environmental data such as temperature and humidity.

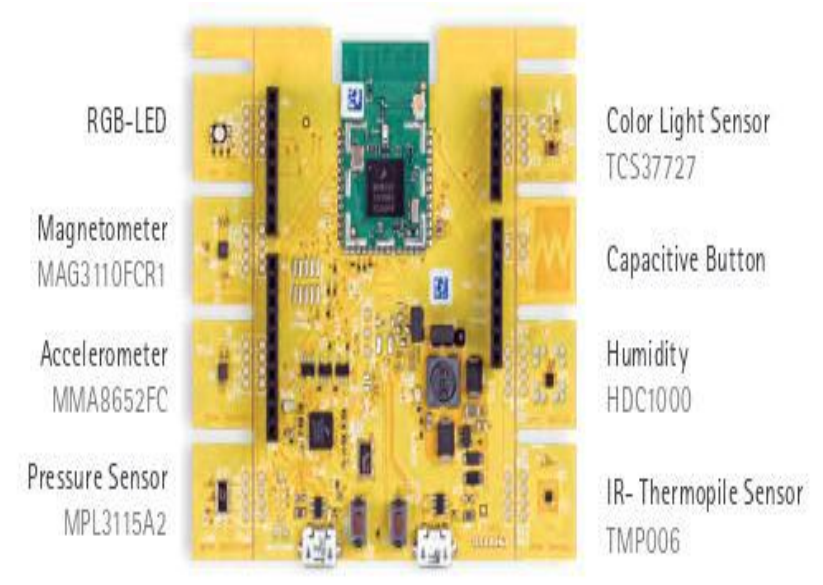

Fig-7: Phynode Board

\section{IMPLEMENTATION}

\subsection{Proposed Method}

In the existing the method sensors are used and additionally in the proposed method e paper technology is used to reduce the power consumption.

\subsubsection{EPaper}

This ePaper is the latest technological advances made by the Industrial Technology Research Institute (ITRI) in flexible displays. epaper are rewritable, environmentally friendly thermal printable e-paper. The epaper, devised to reduce traditional paper consumption.

\subsubsection{Procedure}

Initially develop the ePaper with the help of passive LED's and then insert the 2 sensors in the same phynode-ePaper, 1 is the temperature sensor and 2 is the proximity sensor.

The temperature sensor to monitor the how much temperature in particular place, and proximity sensor is the by using to detect how many persons to visit particular product.

In this project 6loWPAN, PhyGATE-AM335X (gateway board) and MQTT are used.

\subsubsection{Phynode-ePaper}

PhyNODE-ePaper 1 is based on a phyWAVECC2650 and can be integrated through SPI into existing networks BLE or IEEE 802.15.4|6LowPAN. It also has a user-configurable button, temperature sensor as well as a proximity sensor. We designed it with energy efficiency in mind in order to ensure operation with a button cell or a lithium cell in AA format. Therefore, the graphical ePaper display only uses energy during the updating process.

Once the content is displayed, PhyNODE- ePaper 1 can be switched off for a configurable amount of time. Exemplary uses for ePaper Node are configurable name tags, price tags or information items for building automation. 


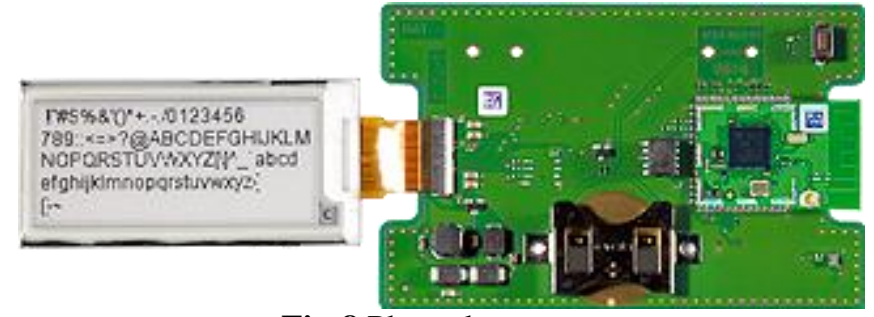

Fig-8 Phynode epaper

\subsubsection{Project Modules}

The below figure shows the block diagram of the complete project which consists of mainly three modules

1. Phynode ePaper

2. WEGA Gateway

3. Mobile/Computer
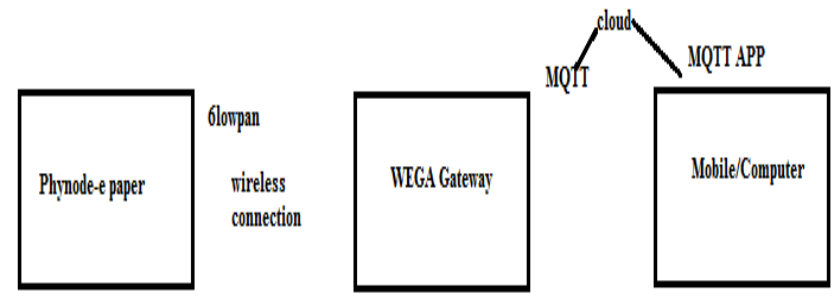

Fig-9 Block Diagram of Monitoring Price Tag Using E Paper

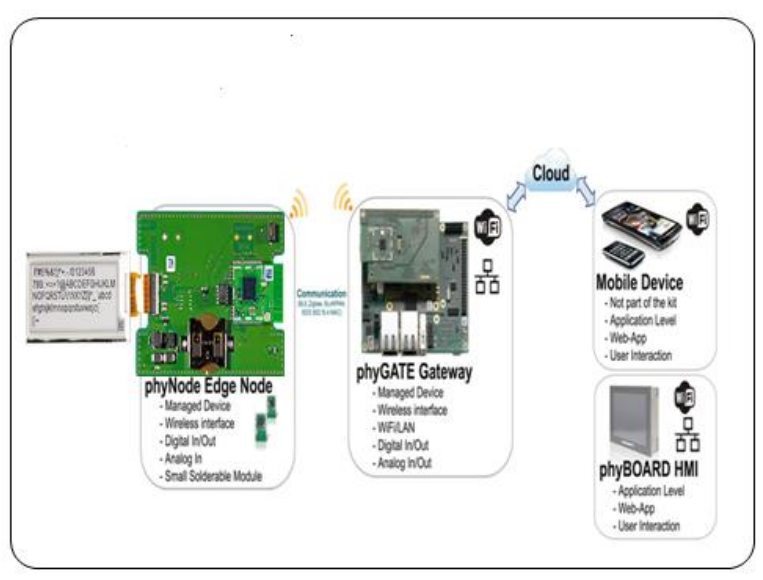

Fig-10 Picture Based Project Implementation

\subsubsection{E-Paper Passive Led Display}

By using passive LED Once the content is displayed in PhyNODE-ePaper.

The graphical ePaper display only uses energy during the updating process. Once the content is displayed, PhyNODEePaper can be switched off for a configurable amount of time.

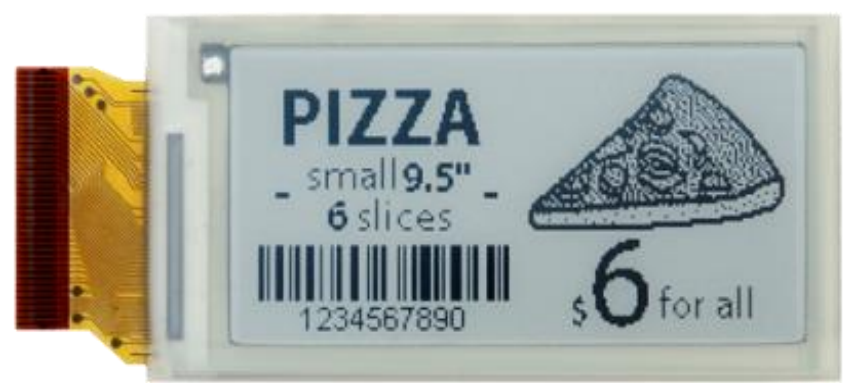

Fig-11 Passive Led ePaper Display

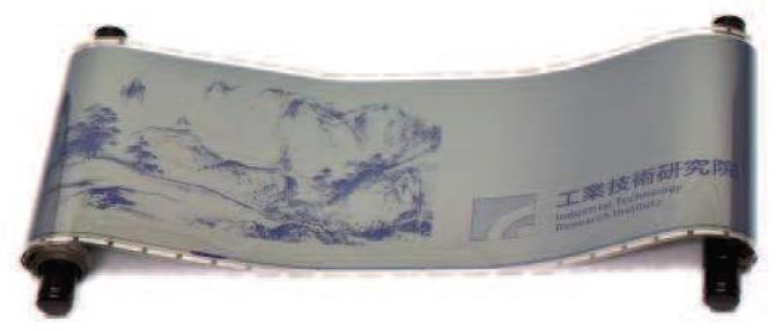

Fig-12 ePaper

\subsubsection{MQTT}

> MQTT(Message Queue Telemetry Transport) is a publish-subscribe based "light weight" messaging protocol for use on top of the TCP/IP protocol .

$>$ Publish-subscribe means when a thing publishes the message in a topic. A topic is like a channel where each thing subscribed to that topic will receive that message. You can imagine this like a Twitter of Things.

$>$ We collect the total data with a MQTT application in mobile, tabs or computers.
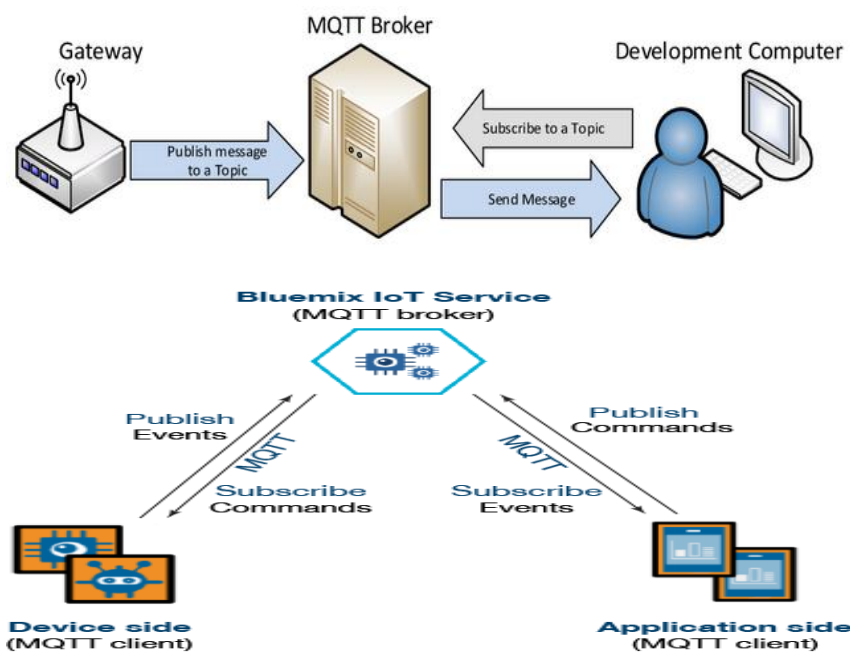

Fig-13 Representation of MQTT

\section{RESULTS}

\subsection{Results}

The main goal of this project was to develop the driver for all the sensors present on the Phynode and develop an application to collect the data from sensor of Phynode and send it to Phygate. 
So the drivers of some sensors are developed successfully and to see the results one demo application is develop which is sending the Phynode data to the Phygate.

After the compilation of all the developed drivers and application we get the binary Image of Contiki. We ported that binary Image to the Phynode using the Open OCD debugger. After the porting of the Contiki OS on the Phynode, it started booting and collecting the data by using sensors and start advertising the data to the air. Image of the advertising node is shown below in Fig below. The indication of the advertising is the RGB Led which glows to advertise the data.

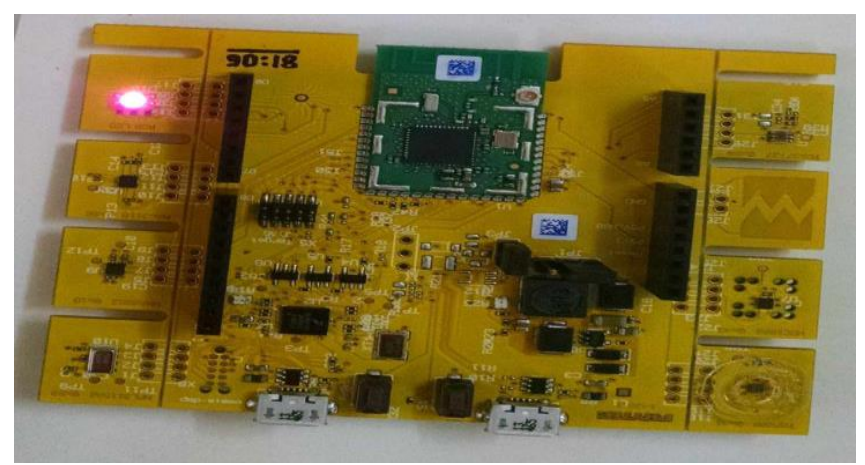

Fig-15 Advertising using RGB LED

For showing the results one prebuilt python script is used at phygate. python script integrate 6LoWPAN Protocol and converts the received raw Data of sensors and show the result on the console of the phygate.

The screenshots of the received data is shown in Fig

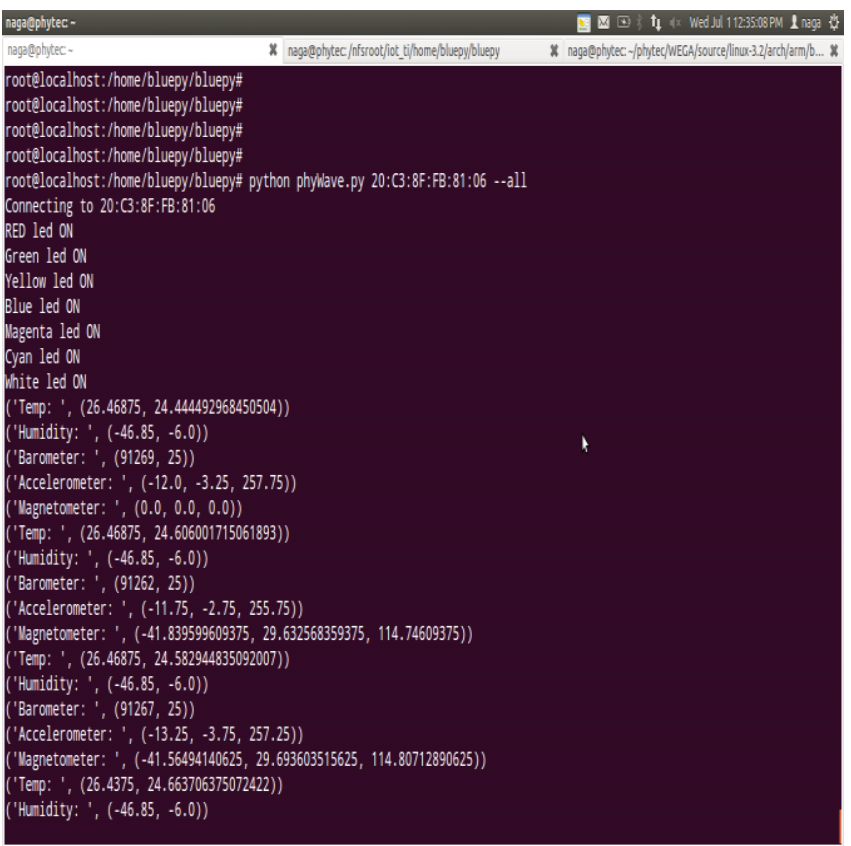

Fig-16 Screenshot of the received data using Python scripts

The results of some sensors data using the android application whose screenshots are shown below:

\subsection{Input Images}

The data is given or write by using android app

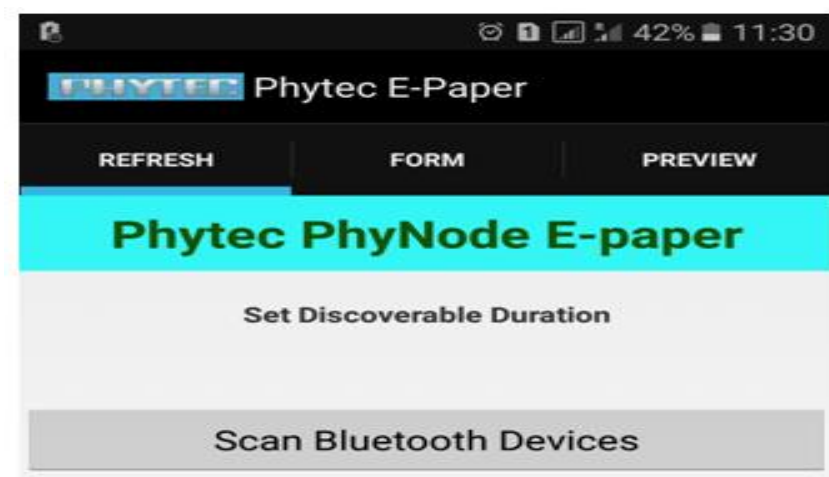

Fig-17 Input Image For Phytec ePaper

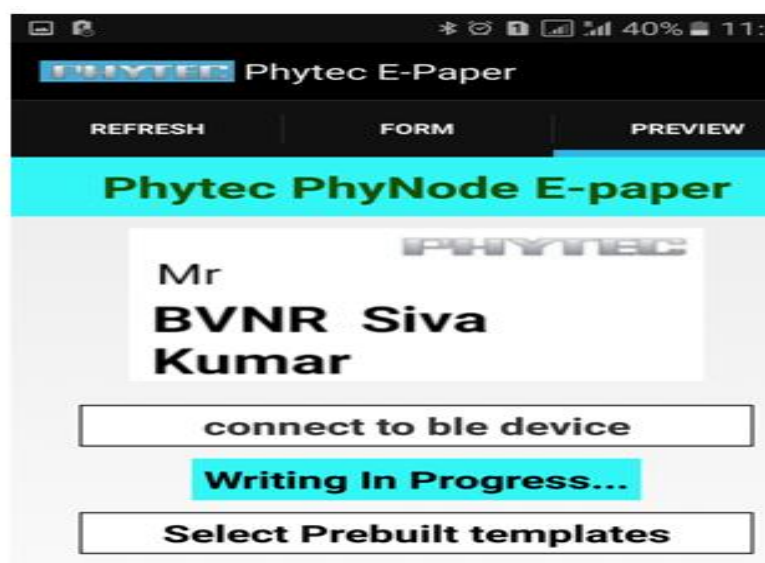

Fig -18 Input Images with Writing in Progress

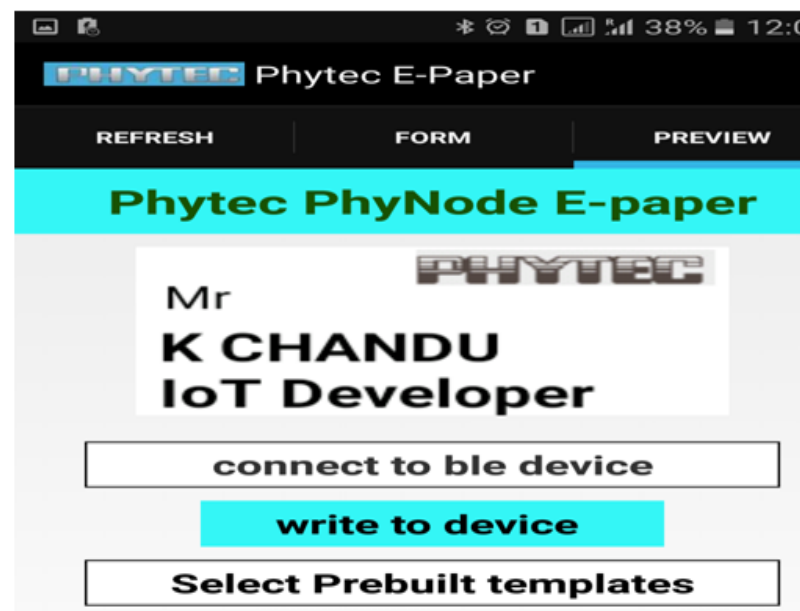

Fig-19 Input Image Write To Device

\subsection{Output Images}

The below images shows the output results using phytec board. 


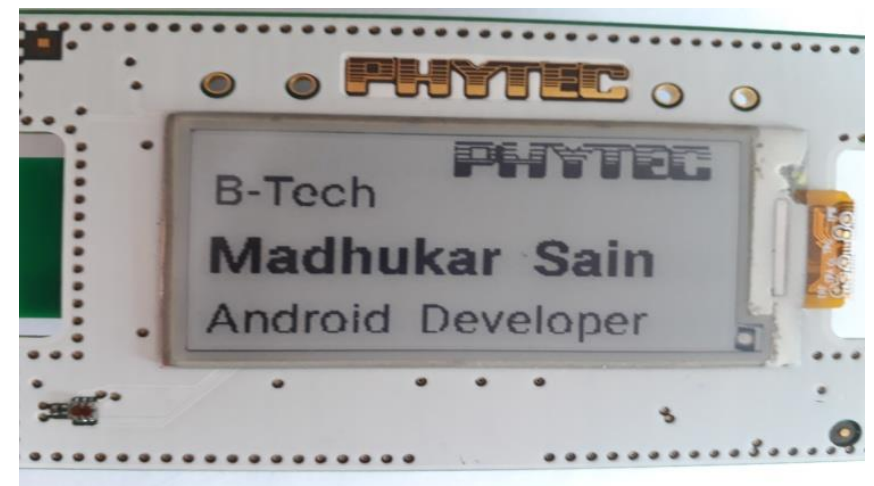

Fig-20 (a) Output Image of ePaper

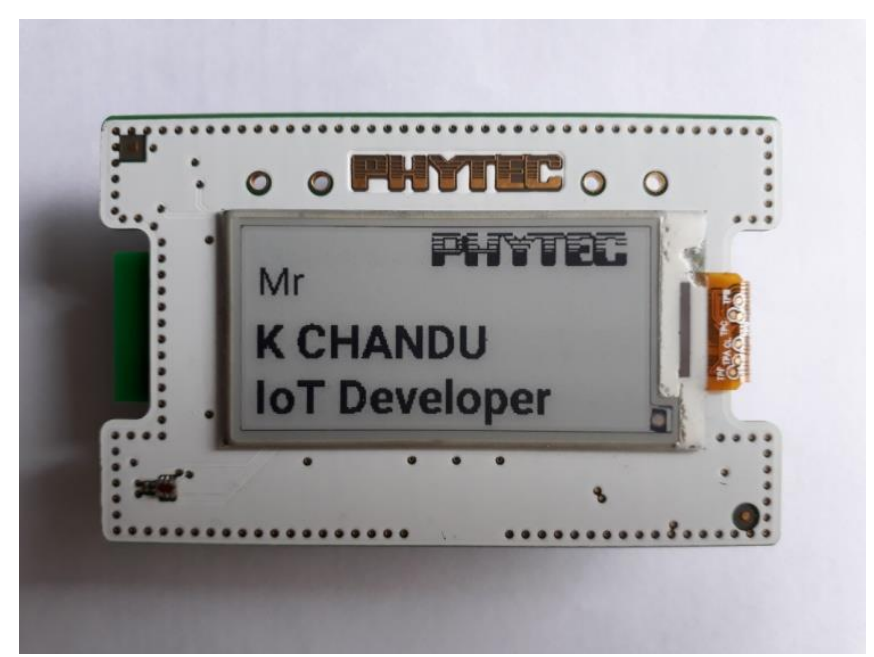

Fig-20 (b) Output Image of ePaper

\subsection{Output Images of Sensors}

The temperature, humidity, accelerometer and light sensor data are shown in below images

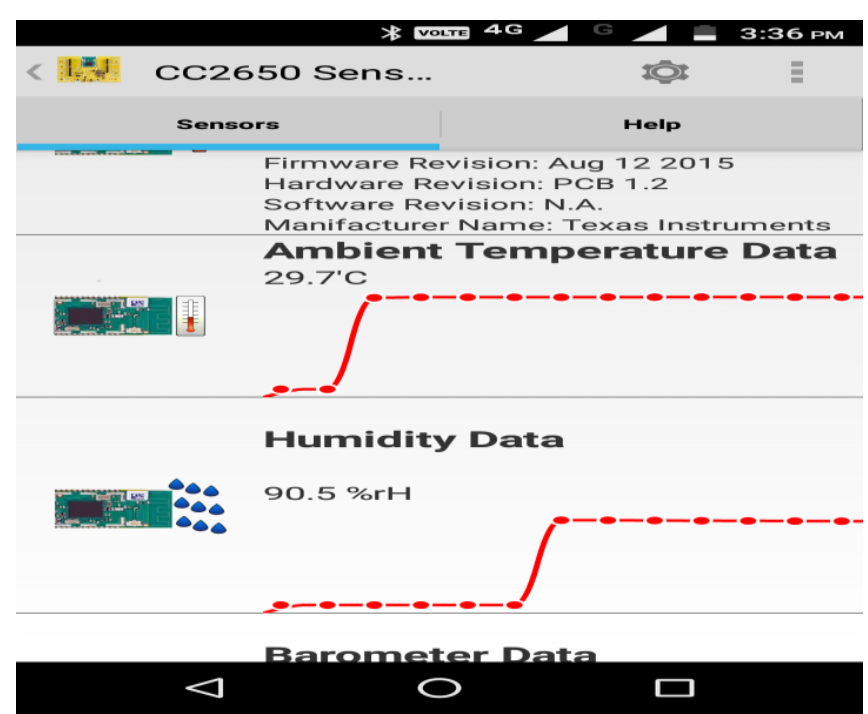

Fig-21 Data of Temperature And Humidity

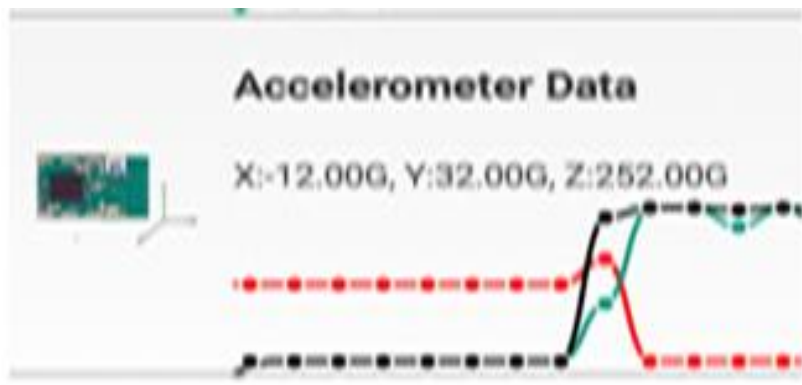

Fig-22 Data of Accelerometer

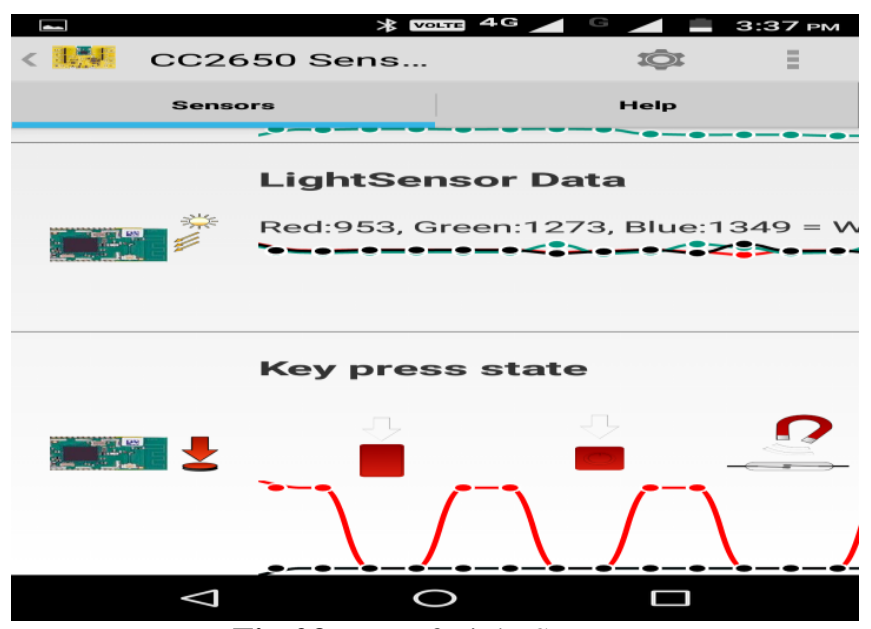

Fig-23 Data of Light Sensor

\section{COMPARISON GRAPH}

Figure below shows the power led supply which has heavy power lose.

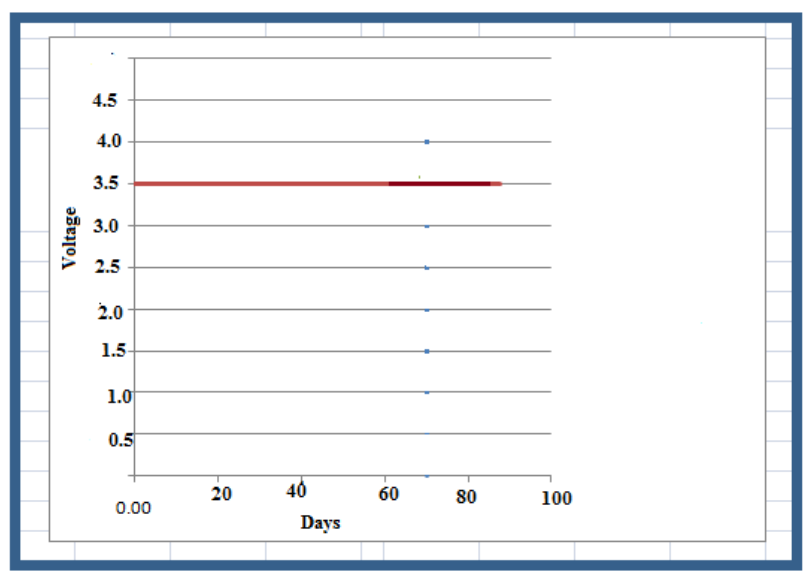

Fig-20 Power Led Display

Figure below shows the passive led display which has very low power lose. 


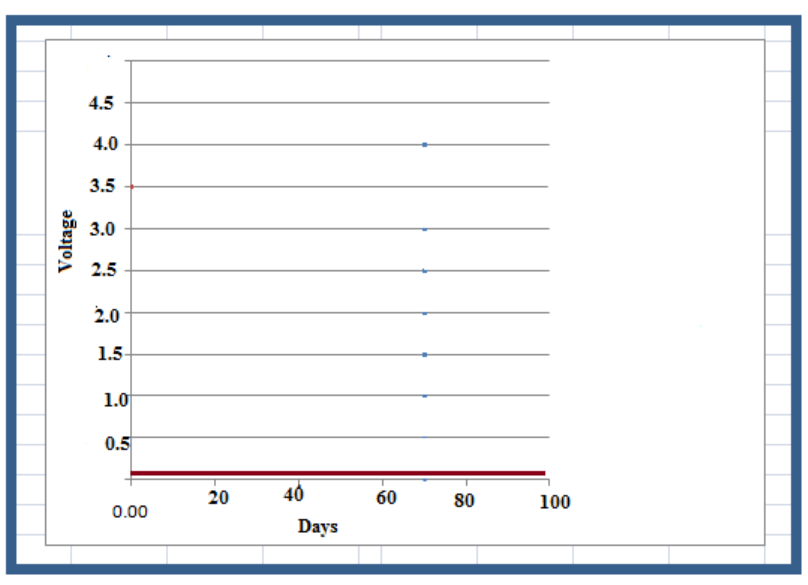

Fig-21 Passive Led Display

\section{CONCLUSION}

From the project result it is very useful in supermarkets, shopping malls and etc. and to develop phynode epaper based on IoT technology using 6loWPAN, MQTT and phyGATEAM335x this things. The same time we can write and read the data in epaper display. And also only read the data of temperature sensor and proximity sensor.

\section{FUTURE SCOPE}

To adding the different sensors in price tag automatically to analyse the surrounds. By using ePaper we can develop flexible products mobile and TV display. E-banners and esignage. E-cards, E-badge, E-tags, E-tickets. We can develop with wireless connections this total products based on IoT.

\section{REFERENCES}

[1]. R. Hinden and S. Deering. IP Version 6 Addressing Architecture. RFC 4291, Internet Engineering Task Force, February 2006.

[2]. S. Thomson, T. Narten, and T. Jinmei. IPv6 Stateless Address Autoconfiguration. RFC 4862, Internet Engineering Task Force, September 2007.

[3]. Pallàs-Areny R., "Section 2 Sensing methods and sensors", Electronic Instrumentation course, (2013).

[4]. LeHong H., Fenn J., Leeb-du Toit R., "Hype Cycle for Emerging Technologies", Gartner, Worldwide, July 2014.

[5]. Friedemann Mattern and Christian Floerkemeier. From the Internet of Computers to the Internet of Things, volume 6462 of LNCS, pages 242-259. Springer, 2010.

[6]. R. Braden. Requirements for Internet Hosts - Application and Support. RFC 1123, Internet Engineering Task Force, October 1989.

[7]. R. Braden. Requirements for Internet Hosts Communication Layers. RFC 1122, Internet Engineering Task Force, October 1989.

[8]. J. Postel. Internet Protocol. RFC 0791, Internet Engineering Task Force, September 1981.

[9]. Postel, J. (1980). User Datagram Protocol. http://tools.ietf.org/html/rfc0768.

[10]. IEEE Standard for Information TechnologyTelecommunications and Information Exchange Between
Systems- Local and Metropolitan Area Networks- Specific Requirements Part 3

[11]. S. Thomson, T. Narten, and T. Jinmei. IPv6 Stateless Address Autoconfiguration. RFC 4862, Internet Engineering Task Force, September 2007.

[12].http://zolertia.sourceforge.net/wiki/index.php/Mainpage :Contiki_Lesson_0

[13]. Contiki 2.x Reference Manual Generated by Doxygen 1.4.1.

[14]. A Trek Through the Contiki Operating System created by Hossam Ashtawy, Troy Brown, Xiaojun Wang, Yuan Zhang Department of Computer Science and Engineering Michigan State University

[15]. A. Dunkels, B. Gronvall and T. Voigt, -Contiki - A Lightweight and Flexible Operating System for Tiny Networked Sensorsll, Swedish Institute of Computer Science, 2004 